NOTICE: this is the author's version of a work that was accepted for publication in Automatica. Changes resulting from the publishing process, such as peer review, editing, corrections, structural formatting, and other quality control mechanisms may not be reflected in this document. Changes may have been made to this work since it was submitted for publication. A definitive version was subsequently published in Automatica and its Applications [VOL41, ISSUE8, 2005] http://dx.doi.org/10.1016/j.automatica.2005.01.018 


\title{
A parametrization of the solutions of the finite-horizon LQ problem with general cost and boundary conditions *
}

\author{
Augusto Ferrante $^{\mathrm{a}}$, Giovanni Marro ${ }^{\mathrm{b}}$, Lorenzo Ntogramatzidis ${ }^{\mathrm{b}}$ \\ a Dipartimento di Ingegneria dell' Informazione, Università di Padova, via Gradenigo, 6/B - 35131 Padova, Italy \\ ${ }^{\mathrm{b}}$ Dipartimento di Elettronica, Informatica e Sistemistica, Università degli Studi di Bologna, viale Risorgimento, 2 - 40136
} Bologna, Italy

\begin{abstract}
A generalization of the finite-horizon linear quadratic regulator problem is proposed for LTI continuous-time controllable systems. In particular, a formulation of the LQ problem is considered, with affine constraints on the initial and the terminal states and with general quadratic costs in the initial and terminal states. The solution presented is simple and attractive from a computational point of view, and it is based on the solutions of an algebraic Riccati equation and of a Lyapunov equation, that enable all the solutions of the Hamiltonian differential equation to be parametrized in closed form.
\end{abstract}

Key words: Finite-horizon LQ problem, Hamiltonian differential equation, algebraic Riccati equations, boundary conditions, optimal cost.

\section{Introduction}

The linear quadratic regulator (LQR) is one of the fundamental and deeply investigated problems in optimal control theory. In particular, the solution of the finitehorizon LQ problem, in which the initial state is assigned and the terminal state is weighted quadratically in the performance index, is traditionally expressed in terms of the solution of a matrix Riccati differential equation (see e.g. [9], [3]), whose integration, usually based on finite difference methods, leads to a heavy computational burden.

In recent years, different kinds of extensions of the standard LQ problem have been investigated, taking into account constraints on the initial and terminal states, or on the whole state trajectory or control law, see e.g. [2], [6], [7], [8], [13] and references therein. In particular, many valuable contributions on the so-called point-topoint version of the finite-horizon LQ problem, in which

\footnotetext{
* This paper was not presented at any IFAC meeting. Partially supported by the ministry of higher education of Italy (MIUR), under project Identification and Control of Industrial Systems. Corresponding author L. Ntogramatzidis.

Email addresses: augusto@dei.unipd.it (Augusto Ferrante), gmarro@deis.unibo.it (Giovanni Marro), Intogramatzidis@deis. unibo.it (Lorenzo Ntogramatzidis).
}

the initial and the terminal states (or the corresponding outputs) are assigned have been presented in the most recent literature (see e.g. [11] and references therein). The problem considered in this paper is the LQ-optimal finite-horizon control of a LTI controllable system whose initial and terminal states are constrained to belong to affine varieties. Moreover, the finite-horizon quadratic performance index includes an initial and terminal costs that weight the difference between the initial and terminal states and two assigned target states. This formulation of the finite horizon LQ is quite general, since it encompasses the standard case as well as the point-topoint LQ.

We shall show that the aforementioned problem can be solved with the tools that are currently employed for the solution of the infinite-horizon LQ regulator, i.e., the algebraic Riccati equation and the algebraic Lyapunov equation. In particular, the stabilizing and the anti-stabilizing solutions of a single algebraic Riccati equation yield an explicit representation of all the statecostate solutions of the Hamiltonian differential equation in terms of two parameters. Their values are computed in terms of the specified boundary conditions.

In this context, new closed-form expressions of the optimal value of the performance index are also derived. In the special cases mentioned above, in which the extreme states are assigned or either is fixed and the other is weighted quadratically, the optimal cost can be ex- 
pressed as a quadratic form of the assigned state(s). The solution presented yields efficient computational algorithms based on strict algebraic tools. In fact, both the optimal state trajectory and the corresponding control law are expressed as functions of two matrix exponentials, whose exponents are stable in the overall time interval, thus ensuring the robustness of the solution proposed even for large time horizons.

This paper is organized as follows. In Section 2, the finite-horizon LQ problem with general quadratic cost and affine constraints on the extreme states is stated. Then, in Section 3, a parametrization of the solutions of the Hamiltonian differential equation involving the extreme solutions of the associated ARE is presented. In Section 4, a detailed analysis on the feasibility of the Pontryagin equations for the optimal control problem herein considered is carried out. Moreover, it is shown that the optimal cost can be expressed in closed form as a function of the problem's data. Furthermore, it is proved that the Pontryagin equations are necessary and sufficient in the considered case, and they guarantee the existence of an optimal solution. Finally, in Section 5, some important special cases are taken into account: the point-to-point LQ and the classical finite-horizon LQ. In particular, some classical results of optimal control theory are derived in a simple and clear form.

Notation. Throughout this paper, the symbol $\mathbb{R}^{n \times m}$ will denote the space of $n \times m$ real matrices. The image and the null-space of matrix $A$ are denoted by $i m A$ and $\operatorname{ker} A$, whereas $A^{\top}$ and $A^{+}$denote the transpose and the Moore-Penrose pseudoinverse of $A$, respectively. The symbol $I_{n}$ stands for the $n \times n$ identity matrix.

\section{Statement of the problem}

Consider the linear time-invariant state differential equation

$\dot{x}(t)=A x(t)+B u(t)$,

where, for all $t \geq 0, x(t) \in \mathbb{R}^{n}$ is the state, $u(t) \in \mathbb{R}^{m}$ is the control input, $A$ and $B$ are real constant matrices of proper sizes.

Let $t_{f}>0$. Consider two full-row rank matrices $W_{0} \in \mathbb{R}^{s \times n}, \quad W_{f} \in \mathbb{R}^{r \times n}$ and two vectors $w_{0} \in \mathbb{R}^{s}$, $w_{f} \in \mathbb{R}^{r}$ with $s, r \leq n$. Consider two functions $\chi_{0}: \mathbb{R}^{n} \rightarrow$ $\mathbb{R}^{s}$ and $\chi_{f}: \mathbb{R}^{n} \rightarrow \mathbb{R}^{r}$ that represent the affine constraints on the initial and terminal states ${ }^{1}$ of (1) ex-

\footnotetext{
1 Notice that these constraints encompass the case when the initial and terminal values $y(0)$ and $y\left(t_{f}\right)$ of an output $y(t)=C x(t)$ of the dynamical system described by (1) are assigned. In fact in this case the corresponding constraints for the state are expressed by (2) with $W_{0}=W_{f}=C, w_{0}=y(0)$ and $w_{f}=y\left(t_{f}\right)$.
}

pressed by

$$
\begin{aligned}
& \chi_{0}(x(0)):=W_{0} x(0)-w_{0}=0, \\
& \chi_{f}\left(x\left(t_{f}\right)\right):=W_{f} x\left(t_{f}\right)-w_{f}=0 .
\end{aligned}
$$

Let $\Pi:=\left[\begin{array}{cc}Q & S \\ S^{\top} & R\end{array}\right]=\Pi^{\top} \geq 0$, with $Q \in \mathbb{R}^{n \times n}$, $S \in \mathbb{R}^{n \times m}$ and $R \in \mathbb{R}^{m \times m}$. We assume $R>0$ and define the Schur complement $\tilde{Q}:=Q-S R^{-1} S^{\top}$ that is clearly positive semidefinite. Let $P_{0}, P_{f} \in \mathbb{R}^{n \times n}$ be symmetric and positive semidefinite, let $z_{0}, z_{f} \in \mathbb{R}^{n}$, and eventually define the functions $\beta_{0}, \beta_{f}: \mathbb{R}^{n} \rightarrow \mathbb{R}$ as

$$
\begin{aligned}
& \beta_{0}(x):=\left(x-z_{0}\right)^{\top} P_{0}\left(x-z_{0}\right), \\
& \beta_{f}(x):=\left(x-z_{f}\right)^{\top} P_{f}\left(x-z_{f}\right) .
\end{aligned}
$$

The optimal control problem dealt with in this paper can be stated as follows.

Problem 1 Find a measurable input function $u(t)$, $t \in\left[0, t_{f}\right]$, and an absolutely continuous state trajectory $x(t), t \in\left[0, t_{f}\right]$, minimizing the quadratic performance index

$$
\begin{aligned}
& J(x, u):=\beta_{0}(x(0))+\int_{0}^{t_{f}}\left[x^{\top}(t) Q x(t)+2 x^{\top}(t) S u(t)\right. \\
&\left.+u^{\top}(t) R u(t)\right] d t+\beta_{f}\left(x\left(t_{f}\right)\right),
\end{aligned}
$$

under the constraints (1) and (2).

\section{Hamiltonian state-costate trajectories}

Refer to Problem 1. By following the same procedure carried out in $[9, \mathrm{p} .133]$, since the constraints are affine, it is found that if $u(t)$ and $x(t), t \in\left[0, t_{f}\right]$, are optimal for Problem 1, then there exist $\lambda:\left[0, t_{f}\right] \rightarrow \mathbb{R}^{n}, \nu \in \mathbb{R}^{s}$ and $\mu \in \mathbb{R}^{r}$ such that $x(t), \lambda(t), u(t), \nu$ and $\mu$ satisfy the following equations:

$$
\begin{aligned}
\dot{x}(t)= & A x(t)+B u(t), \\
& \chi_{0}(x(0))=0, \quad \chi_{f}\left(x\left(t_{f}\right)\right)=0, \\
\dot{\lambda}(t)= & -Q x(t)-S u(t)-A^{\top} \lambda(t), \\
& \lambda(0)=-P_{0} x(0)+P_{0} z_{0}-W_{0}^{\top} \nu, \\
& \lambda\left(t_{f}\right)=P_{f} x\left(t_{f}\right)-P_{f} z_{f}+W_{f}^{\top} \mu, \\
R u(t)+ & S^{\top} x(t)+B^{\top} \lambda(t)=0 .
\end{aligned}
$$

Since $R>0$, equation (9) can be solved in $u(t)$ yielding

$u(t)=-R^{-1}\left(S^{\top} x(t)+B^{\top} \lambda(t)\right)$. 
Hence, by (4) and (6) it follows that $\left[x^{\top}(t) \lambda^{\top}(t)\right]^{\top}$ is a solution of the Hamiltonian differential equation

$\left[\begin{array}{l}\dot{x}(t) \\ \dot{\lambda}(t)\end{array}\right]=H\left[\begin{array}{l}x(t) \\ \lambda(t)\end{array}\right]$

where $H:=\left[\begin{array}{cc}A-B R^{-1} S^{\top} & -B R^{-1} B^{\top} \\ -Q+S R^{-1} S^{\top} & -A^{\top}+S R^{-1} B^{\top}\end{array}\right]$ is

called the Hamiltonian matrix. We make the following standing assumptions.

(A1) the pair $(A, B)$ is controllable

(A2) $H$ has no eigenvalues on the imaginary axis

Under assumptions (A1)-(A2), the continuous-time algebraic Riccati equation (CARE)

$P A+A^{\top} P-(S+P B) R^{-1}\left(S^{\top}+B^{\top} P\right)+Q=0$

has a maximal solution $P_{+}=P_{+}^{\top} \geq 0$ and a minimal solution $P_{-}=P_{-}^{\top} \leq 0$ such that the gap matrix $\Delta:=P_{+}-P_{-}$is positive definite (see $[10$, Lemma 7 and proof of Theorem 6] for details), and the eigenvalues of the closed-loop matrices

$A_{+}:=A-B K_{+} \quad$ and $\quad A_{-}:=A-B K_{-}$,

where $K_{+}$and $K_{-}$are defined as

$K_{+}:=R^{-1}\left(S^{\top}+B^{\top} P_{+}\right), K_{-}:=R^{-1}\left(S^{\top}+B^{\top} P_{-}\right)$,

are stable and anti-stable, respectively. Hence, $P_{+}$and $P_{-}$are called the stabilizing and anti-stabilizing solutions of the CARE (12).

Remark 1 The matrices $P_{+}$and $A_{+}$may be computed by standard and reliable numerical algorithms available in any control package. Concerning $P_{-}$, it is well-known (see $\left[10\right.$, p.354]) that $\Delta^{-1}$ satisfies the Lyapunov equation

$A_{+} \Delta^{-1}+\Delta^{-1} A_{+}^{\top}+B R^{-1} B^{\top}=0$.

Hence, we may solve (15) and we get $P_{-}=P_{+}-\Delta$.

The following theorem provides an explicit formula parametrizing all the solutions of the Hamiltonian differential equation.

Theorem 1 Let assumptions (A1)-(A2) hold. The set of solutions of the Hamiltonian differential equation (11) in $\left[0, t_{f}\right]$ is parametrized in $p, q \in \mathbb{R}^{n}$ by

$\left[\begin{array}{l}x(t) \\ \lambda(t)\end{array}\right]=\left[\begin{array}{c}I_{n} \\ P_{+}\end{array}\right] e^{A_{+} t} p+\left[\begin{array}{c}I_{n} \\ P_{-}\end{array}\right] e^{A_{-}\left(t-t_{f}\right)} q$.
Proof: Let $J_{+}:=\left[\begin{array}{ll}I_{n} & P_{+}\end{array}\right]^{\top}$ and $J_{-}:=\left[\begin{array}{ll}I_{n} & P_{-}\end{array}\right]^{\top}$. A direct computation shows that $H J_{+}=J_{+} A_{+}$and $H J_{-}=J_{-} A_{-}$(see e.g. [12, Property 4.1.5]). Hence, the family of trajectories (16) satisfies the Hamiltonian differential equation (11) for all $p$ and $q$.

Conversely, since (11) is a linear differential equation of order $2 n$, it is sufficient to show that among the linear space of trajectories described by (16), we may select $2 n$ linearly independent ones. To this aim, let $\pi_{i}:=\left[\begin{array}{ll}p_{i}^{\top} & q_{i}^{\top}\end{array}\right]^{\top} \in \mathbb{R}^{2 n}, i=1, \ldots, 2 n$, be linearly independent, and denote by $v_{i}(t):=\left[x_{i}^{\top}(t) \lambda_{i}^{\top}(t)\right]^{\top}$ the corresponding state and costate trajectories given by (16). It follows that $\sum_{i=0}^{2 n} k_{i} v_{i}(t)=0$ for all $t \in\left[0, t_{f}\right]$ implies $\Xi(t) \sum_{i=0}^{2 n} k_{i} \pi_{i}=0$ for all $t \in\left[0, t_{f}\right]$, where $\Xi(t):=\left[J_{+} e^{A_{+} t} J_{-} e^{A_{-}\left(t-t_{f}\right)}\right]$. Notice that $\Xi(t)$ is full rank for all $t \in\left[0, t_{f}\right]$. In fact, if $\xi:=\left[\begin{array}{ll}\xi_{1}^{\top} & \xi_{2}^{\top}\end{array}\right]^{\top}$ belongs to the null-space of $\Xi\left(t^{*}\right)$, for $t^{*} \in\left[0, t_{f}\right]$, it follows that $J_{+} e^{A_{+} t^{*}} \xi_{1}=J_{-} e^{A_{-}\left(t^{*}-t_{f}\right)} \xi_{2}$. Since $i m J_{+}$and $i m J_{-}$ are $n$-dimensional $H$-invariant subspaces and the eigenvalues of $H$ restricted to $i m J_{+}$and to $i m J_{-}$are the eigenvalues of $A_{+}$and $A_{-}$, respectively, and since the intersection of two $H$-invariant subspaces is $H$-invariant, it follows that $\mathrm{im} J_{+} \cap \mathrm{im} J_{-}=\{0\}$. Hence, $e^{A_{+} t^{*}} \xi_{1}=0$ and $e^{A_{-}\left(t^{*}-t_{f}\right)} \xi_{2}=0$. It follows that $\xi_{1}=\xi_{2}=0$ since for all $t \in\left[0, t_{f}\right]$ the exponential matrices $e^{A_{+} t}$ and $e^{A_{-}\left(t-t_{f}\right)}$ are non-singular. As a consequence, $k_{i}=0$ for all $i=1, \ldots, 2 n$, since $\pi_{1}, \pi_{2}, \ldots, \pi_{2 n}$ and linearly independent.

\section{Derivation of the optimal control}

In Theorem 1 it has been shown that under assumptions (A1)-(A2) the extreme solutions $P_{+}$and $P_{-}$of the CARE (12) yield an explicit representation of all the solutions of the Hamiltonian differential equation (11) in terms of two parameters $p$ and $q$. In this section we show that equations (4)-(9) admit solutions, and we parametrize the set of such solutions.

Define $F_{+}:=e^{A_{+} t_{f}}$ and $F_{-}:=e^{A_{-} t_{f}}$. By (16), the equations of the constraints (2) lead to

$W_{0} p+W_{0} F_{-}^{-1} q=w_{0}$,

$W_{f} F_{+} p+W_{f} q=w_{f}$.

From (7) and (16) it follows that

$-P_{0}\left(p+F_{-}^{-1} q\right)+P_{0} z_{0}-W_{0}^{\top} \nu=P_{+} p+P_{-} F_{-}^{-1} q$.

Let $V_{0}$ be a basis matrix of $k e r W_{0}$. The former yields

$V_{0}^{\top}\left(P_{+}+P_{0}\right) p+V_{0}^{\top}\left(P_{0}+P_{-}\right) F_{-}^{-1} q-V_{0}^{\top} P_{0} z_{0}=0$. 
The same procedure can be applied to (8) and (16), so as to obtain

$V_{f}^{\top}\left(P_{+}-P_{f}\right) F_{+} p-V_{f}^{\top}\left(P_{f}-P_{-}\right) q+V_{f}^{\top} P_{f} z_{f}=0$,

where $V_{f}$ is a basis matrix of ker $W_{f}$. Equations (17), (18), (19) and (20) yield the following compact expression

$h=N \pi$

where $\pi:=\left[p^{\top} q^{\top}\right]^{\top}$ and

$h:=\left[\begin{array}{c}w_{0} \\ w_{f} \\ V_{0}^{\top} P_{0} z_{0} \\ V_{f}^{\top} P_{f} z_{f}\end{array}\right], N:=\left[\begin{array}{cc}W_{0} & W_{0} F_{-}^{-1} \\ W_{f} F_{+} & W_{f} \\ V_{0}^{\top}\left(P_{0}+P_{+}\right) & V_{0}^{\top}\left(P_{0}+P_{-}\right) F_{-}^{-1} \\ V_{f}^{\top}\left(P_{f}-P_{+}\right) F_{+} & V_{f}^{\top}\left(P_{f}-P_{-}\right)\end{array}\right]$.

If $s=n$ (resp. $r=n$ ), the constraints (2) completely assign the initial (resp. terminal) state and the third (resp. fourth) block-row of $N$ and $h$ disappears.

So far, we have shown that (21) follows from (4)-(9). Notice that also the converse is true. In fact, if $p$ and $q$ are solutions of (21), equations (16) and (10) enable $x(t)$, $\lambda(t)$ and $u(t)$ to be computed. Finally, vectors $\nu$ and $\mu$ may be computed from equations (7) and (8), since $W_{0}$ and $W_{f}$ are full-row rank.

The results that follow show that the linear system (21), and hence the set of equations (4)-(9), is feasible, and in Section 4.1 it will be proved that all its solutions give rise to state trajectories and control laws yielding the same cost.

Lemma 1 The following results hold.

(1) The matrix $\Omega:=\Delta^{-1}-F_{+} \Delta^{-1} F_{+}^{\top}$ is positive definite.

(2) The matrix $\Sigma:=I_{n}-F_{+} F_{-}^{-1}$ satisfies $\Sigma=\Omega \Delta$, so that it is non-singular.

(3) The matrix $L:=\left[\begin{array}{cc}I_{n} & F_{-}^{-1} \\ F_{+} & I_{n}\end{array}\right]$ is non-singular and its inverse is given by

$L^{-1}=\left[\begin{array}{cc}F_{-}^{-1} \Sigma^{-1} F_{-} & -F_{-}^{-1} \Sigma^{-1} \\ -\Sigma^{-1} F_{+} & \Sigma^{-1}\end{array}\right]$.

(4) The matrix $\Theta:=\Omega^{-1}-P_{+}-\Omega^{-1} F_{+}\left(P_{+}+\right.$ $\left.F_{+}^{\top} \Omega^{-1} F_{+}\right)^{-1} F_{+}^{\top} \Omega^{-1}$ is positive semidefinite, and is positive definite if $\tilde{Q}:=Q-S R^{-1} S^{\top}$ is such.
Proof: First, by [14, Theorem 5], it is found that $\Delta^{-1}=\int_{0}^{+\infty} e^{A_{+} t} B R^{-1} B^{\top} e^{A_{+}^{\top} t} d t$. As a consequence,

$$
\begin{aligned}
\Omega & =\Delta^{-1}-\int_{0}^{+\infty} e^{A_{+}\left(t+t_{f}\right)} B R^{-1} B^{\top} e^{A_{+}^{\top}\left(t+t_{f}\right)} d t \\
& =\int_{0}^{t_{f}} e^{A_{+} t} B R^{-1} B^{\top} e^{A_{+}^{\top} t} d t
\end{aligned}
$$

which is the closed-loop reachability grammian on $\left[0, t_{f}\right]$ and is positive definite by assumption (A1). From [14, Lemma 8], $A_{+}=-\Delta^{-1} A_{-}^{\top} \Delta$, hence $F_{-}^{-1}=\Delta^{-1} F_{+}^{\top} \Delta$. A straightforward computation shows that $\Sigma=I_{n}-F_{+} \Delta^{-1} F_{+}^{\top} \Delta=\Omega \Delta$. It follows that $\Sigma$ is non-singular. Moreover, the determinant of $L$ equals that of $\Sigma$. Hence, $L$ is non-singular as well. The expression of $L^{-1}$ follows after simple algebraic manipulations. Assume $\tilde{Q}>0$. Then, from $P_{+} \geq 0$ and

$$
P_{+} A_{+}+A_{+}^{\top} P_{+}+P_{+} B R^{-1} B^{\top} P_{+}+\tilde{Q}=0
$$

which is a direct consequence of the CARE (12), it follows that $P_{+}>0$ and

$$
A_{+} P_{+}^{-1}+P_{+}^{-1} A_{+}^{\top}+B R^{-1} B^{\top}+\Lambda_{+}=0
$$

with $\Lambda_{+}:=P_{+}^{-1} \tilde{Q} P_{+}^{-1}>0$. Let $\Phi:=P_{+}^{-1}-\Delta^{-1}$. From (15) and (22) we get $A_{+} \Phi+\Phi A_{+}^{\top}+\Lambda_{+}=0$, that yields $\Phi>0$. Moreover, the same argument used to prove that $\Omega>0$ now shows that $\Phi-F_{+} \Phi F_{+}^{\top}>0$. Hence $P_{+}^{-1}>\Delta^{-1}+F_{+} \Phi F_{+}^{\top}$. It follows that

$$
\begin{aligned}
P_{+} & <\left(\Delta^{-1}+F_{+} \Phi F_{+}^{\top}\right)^{-1}=\left(\Omega+F_{+} P_{+}^{-1} F_{+}^{\top}\right)^{-1} \\
& =\Omega^{-1}+\Omega^{-1} F_{+}\left(-P_{+}-F_{+}^{\top} \Omega^{-1} F_{+}\right)^{\top} F_{+}^{\top} \Omega^{-1}
\end{aligned}
$$

which leads to $\Theta>0$. Let now $\tilde{Q}$ be singular. Consider the new problem obtained by changing the matrix $Q$ with the new one $Q_{\varepsilon}:=Q+\varepsilon I_{n}$, where $\varepsilon \geq 0$. If $\varepsilon>0$, $Q_{\varepsilon}-S R^{-1} S^{\top}$ is positive definite so that, by the previous argument, the corresponding $\Theta$ matrix is positive definite. As it is well known (see [12, Theorem 4.1.7]), $P_{+}$is a continuous function of $\varepsilon$, so that $\Theta$ is such (note, in fact, that $\Omega$ is well defined and positive definite even for $\varepsilon=0$, and the same is true for $\left.P_{+}+F_{+}^{\top} \Omega^{-1} F_{+}\right)$. Therefore, by continuity, $\Theta \geq 0$ for $\varepsilon=0$.

Let $x_{0}, x_{f}$ be solutions of $W_{0} x_{0}=w_{0}$ and $W_{f} x_{f}=w_{f}$, respectively. Then, all vectors $x(0)$ and $x\left(t_{f}\right)$ satisfying (2) can be parametrized as $x(0)=p+F_{-}^{-1} q=V_{0} \sigma+x_{0}$ and $x\left(t_{f}\right)=F_{+} p+q=V_{f} \rho+x_{f}$, where $\sigma \in \mathbb{R}^{s}$ and $\rho \in \mathbb{R}^{r}$ are two new variables accounting for all possible initial and terminal states satisfying the constraints (2). 
As a result, (21) may be written as

$$
\left[\begin{array}{cc}
L & -V \\
V^{\top} G & 0
\end{array}\right]\left[\begin{array}{l}
\pi \\
\tau
\end{array}\right]=\left[\begin{array}{c}
x \\
V^{\top} P z
\end{array}\right]
$$

with $V:=\operatorname{diag}\left(V_{0}, V_{f}\right), P:=\operatorname{diag}\left(P_{0}, P_{f}\right)$ and

$$
\begin{aligned}
& G:=\left[\begin{array}{cc}
P_{0}+P_{+} & \left(P_{0}+P_{-}\right) F_{-}^{-1} \\
\left(P_{f}-P_{+}\right) F_{+} & P_{f}-P_{-}
\end{array}\right], \\
& x:=\left[\begin{array}{l}
x_{0} \\
x_{f}
\end{array}\right], \pi:=\left[\begin{array}{l}
p \\
q
\end{array}\right], \quad \tau:=\left[\begin{array}{l}
\sigma \\
\rho
\end{array}\right], z:=\left[\begin{array}{l}
z_{0} \\
z_{f}
\end{array}\right] .
\end{aligned}
$$

Lemma 2 System (23) admits solutions $\pi, \tau$, for all $x$, and $z$.

Proof: From the identity $F_{-}^{-1} \Sigma^{-1}\left(F_{-}-F_{+}\right)=$ $F_{-}^{-1} \Sigma^{-1}\left(I_{n}-F_{-}^{-1} F_{+}\right) F_{+}^{-1}=I_{n}$ and after simple calculations, it is found that

$G L^{-1}=P+X$

where $\quad X:=\left[\begin{array}{cc}P_{+}+F_{+}^{\top} \Omega^{-1} F_{+} & -F_{+}^{\top} \Omega^{-1} \\ -\Omega^{-1} F_{+} & \Omega^{-1}-P_{+}\end{array}\right]$.

Notice that $X$ is positive semidefinite, since $P_{+}+F_{+}^{\top} \Omega^{-1} F_{+}>0$ and its Schur complement is exactly the matrix $\Theta$ defined in Lemma 1, which was shown to be positive semidefinite.

Equation (23) leads to the relations

$$
\begin{aligned}
& \pi=L^{-1} V \tau+L^{-1} x, \\
& V^{\top}(P+X) V \tau+V^{\top}(P+X) x=V^{\top} P z,
\end{aligned}
$$

that admit at least one solution $(\pi, \tau)$. In fact, there exists a solution $\tau_{x}$ of the equation

$V^{\top}(P+X) V \tau=-V^{\top}(P+X) x$,

since the positive semidefiniteness of $P+X$ yields $i m V^{\top}(P+X) V \supseteq i m V^{\top}(P+X)$. Moreover, there exists a solution $\tau_{z}$ of

$V^{\top}(P+X) V \tau=-V^{\top} P z$,

since $\operatorname{ker} V^{\top}(P+X) V \subseteq \operatorname{ker} P V$. Their difference $\tau_{x}-\tau_{z}$ is a solution of $(27)$.

As already observed, equation (21) is equivalent to (23), so that we may conclude that equation (21) admits solutions. As it is well-known, such solutions are parametrized by

$\pi=N^{+} h+K v$ where $K$ is a basis matrix for $\operatorname{ker} N$ and $v$ is an arbitrary vector.

\subsection{Optimal cost}

The following result provides a simple formula for the computation of the optimal value of the performance index (3) in terms of the initial and terminal values of the state and costate variables.

Lemma 3 Let $x(t)$ and $\lambda(t)$ be a solution of the Hamiltonian differential equation (11), and let $u(t), t \in\left[0, t_{f}\right]$, be given by (10). The following identity holds:

$$
\begin{aligned}
c & :=\int_{0}^{t_{f}}\left[x^{\top}(t) Q x(t)+2 x^{\top}(t) S u(t)+u^{\top}(t) R u(t)\right] d t \\
& =x^{\top}(0) \lambda(0)-x^{\top}\left(t_{f}\right) \lambda\left(t_{f}\right) .
\end{aligned}
$$

Proof: From (10), it is easily found that $c=\int_{0}^{t_{f}} w^{\top}(t) T w(t) d t$ where $w(t):=\left[x^{\top}(t) \lambda^{\top}(t)\right]^{\top}$ and $T:=\operatorname{diag}\left(Q-S R^{-1} S^{\top}, B R^{-1} B^{\top}\right)$. Let now be $U:=\left[\begin{array}{cc}0 & I_{n} \\ I_{n} & 0\end{array}\right]$. A simple computation yields $H^{\top} U+U H=-2 T$, that can be replaced in $c$. By (11)

$$
\begin{aligned}
c & =-\frac{1}{2} \int_{0}^{t_{f}}\left[\dot{w}^{\top}(t) U w(t)+w^{\top}(t) U \dot{w}(t)\right] d t \\
& =-\frac{1}{2}\left[w^{\top}(t) w(t)\right]_{0}^{t_{f}}=x^{\top}(0) \lambda(0)-x^{\top}\left(t_{f}\right) \lambda\left(t_{f}\right) .
\end{aligned}
$$

Corollary 1 Consider Problem 1. Let $p, q \in \mathbb{R}^{n}$, and $\pi:=\left[p^{\top} q^{\top}\right]^{\top}$. Let $x(t)$ and $\lambda(t)$ be the corresponding state and costate trajectories given by (16). Let $u(t)$ be the corresponding input function given by (10). The associated performance index $J(x, u)$ is given by the following quadratic form $\mathfrak{q}(\pi, z)$ in $\pi$ and $z$ :

$J=\mathfrak{q}(\pi, z):=\left[\pi^{\top} z^{\top}\right]\left[\begin{array}{cc}L^{\top}(P+X) L & -L^{\top} P \\ -P L & P\end{array}\right]\left[\begin{array}{l}\pi \\ z\end{array}\right]$.

Proof: The proof follows from (16) and (3).

Corollary 2 All solutions $p$ and $q$ of (21) yield the same cost, which is a positive semidefinite quadratic form of $z_{0}, z_{f}, w_{0}$ and $w_{f}$.

Proof: Let $\left(\pi_{1}, \tau_{1}\right)$ and $\left(\pi_{2}, \tau_{2}\right)$ be two solutions of (23). Denote their difference by $\delta \pi:=\pi_{1}-\pi_{2}$ 
and $\delta \tau:=\tau_{1}-\tau_{2}$. From (27), it follows that $V^{\top}(P+X) V \delta \tau=0$. Then, $\delta \tau \in \operatorname{ker}(P V) \cap \operatorname{ker}(X V)$, since both $P$ and $X$ are positive semidefinite. Hence, (26) yields $L \delta \pi=V \delta \tau \in \operatorname{ker} P \cap \operatorname{ker} X$, so that $\delta \pi \in \operatorname{ker} P L \cap \operatorname{ker} X L$ and then, taking into account $(30)$, it is found that $\mathfrak{q}\left(\pi_{2}, z\right)=\mathfrak{q}\left(\pi_{2}+\delta \pi, z\right)=\mathfrak{q}\left(\pi_{1}, z\right)$, where the last equality is a consequence of the definition of $\delta \pi$. The fact that the optimal cost is a quadratic form of $z_{0}, z_{f}, w_{0}$ and $w_{f}$ is a direct consequence of (21), (30) and Corollary 1.

\subsection{Existence of optimal solutions}

So far, we have considered the necessary conditions expressed by (4)-(9) for Problem 1. In particular, we have proved that (4)-(9) admit solutions, and we have shown that all solutions of (4)-(9) lead to the same cost, i.e., the performance index does not depend on the specific solution of (4)-(9). We hasten to stress that this is not sufficient to guarantee that each solution of (4)-(9) correspond to a solution of Problem 1 . In fact to this aim we still need to show that Problem 1 does admit solutions. Notwithstanding the wealth of results on the existence of solutions of optimal control problems, see e.g. [4, Chapters 7-11], it appears that even the existence issue for Problem 1 is not solved in the literature. The following proposition addresses this issue.

\section{Proposition 1 Problem 1 admits solutions.}

Proof: We first assume that $s=n$, i.e., that $W_{0} \in \mathbb{R}^{n \times n}$, so that the initial state $x(0)$ is completely assigned. In this case the existence of optimal solutions for Problem 1 is proved $^{2}$ in $[1$, p.85]. Therefore, we may conclude that all the solutions of (4)-(9) are indeed solutions of Problem 1 and that the optimal cost is a quadratic form of type (30). To deal with the case when $s<n$, we temporarily add to the original constraints (1) and (2), herein briefly denoted by $\mathfrak{C}$, the additional constraint $\mathfrak{C}_{a}$ given by

$V_{0}^{\top} x(0)=v_{0}$

It is not difficult to see that (2) and (31) assign the whole initial state vector $x(0)$ for any $v_{0} \in \mathbb{R}^{n-s}$. Moreover, we have

$\inf _{\mathfrak{C}} J(x, u)=\inf _{v_{0}}\left[\inf _{\mathfrak{C}, \mathfrak{C}_{a}} J(x, u)\right]$.

The infimum inside square brackets in the right-hand side of (32) has still the form of Problem 1 , where $W_{0}$ is

\footnotetext{
2 Notice that in [1, p.85] matrix $S$ is assumed to be zero. However, the general case when $S$ may differ from zero can be brought back to that of $S=0$ by assigning $u(t)=-$ $R^{-1} S^{\top} x(t)+v(t)$ and by minimizing the cost with respect to the new control variable $v(t)$. Thus, we get an equivalent problem where the corresponding matrix $S$ is zero.
}

replaced by $\left[\begin{array}{ll}W_{0}^{\top} & V_{0}\end{array}\right]^{\top}$ and $w_{0}$ by $\left[\begin{array}{ll}w_{0}^{\top} & v_{0}^{\top}\end{array}\right]^{\top}$, so that we are in the previously considered case of $s=n$. Hence, $\inf _{\mathfrak{C}, \mathfrak{C}_{a}} J(x, u)$ is indeed a minimum. Moreover, in view of Corollary 1 , such minimum is a positive semidefinite quadratic form of $\left[\begin{array}{lllll}v_{0}^{\top} & w_{0}^{\top} & w_{f}^{\top} & z_{0}^{\top} & z_{f}^{\top}\end{array}\right]^{\top}$. Hence, the infimum over $v_{0} \in \mathbb{R}^{n-s}$ in the right-hand side of (32) is a minimum as well. The existence of a minimum of $J(x, u)$ over the original constraints $\mathfrak{C}$ guarantees that Problem 1 has solutions.

As a conclusion, we introduce the following corollary, that points out how equation (28) can be used to determine all the values of the parameters $p$ and $q$ yielding the optimal state, costate and input functions for Problem 1 through (16).

Corollary 3 Let $p$ and $q$ satisfy (28). Then, the corresponding state and costate functions $x(t)$ and $\lambda(t)$ satisfying (16) are optimal for Problem 1. Moreover, the optimal control law can be computed by

$u(t)=-K_{+} e^{A_{+} t} p-K_{-} e^{A_{-}\left(t-t_{f}\right)} q$,

where $K_{+}$and $K_{-}$are defined in (14).

Proof: We have shown that (4)-(9) provide optimal solutions for Problem 1. Now, if $p$ and $q$ satisfy (28), if the corresponding $x(t)$ and $\lambda(t)$ satisfy the Hamiltonian differential equation and $u(t)$ is given by (10), then the state, costate and input functions satisfy (4)-(9) for some $\nu$ and $\mu$, hence they are optimal.

\section{Special cases}

In this section, we consider two particular cases of Problem 1 in which either both the initial and the terminal states are assigned, or the terminal state is weighted in the performance index ${ }^{3}$. In both cases, it will be shown how several classical results concerning the structure of the optimal solution and the optimal cost follow straightforwardly by using the proposed approach, with the further advantage that the dipendence of the optimal state and control and of the optimal cost with the assigned state(s) is herein explicited in closed form. First, we consider the case of sharply assigned initial and final states.

Problem 1a Find a measurable input function $u(t)$, $t \in\left[0, t_{f}\right]$, and an absolutely continuous state trajectory $x(t), t \in\left[0, t_{f}\right]$, minimizing the quadratic performance index (3) with $\beta_{0}=\beta_{f}=0$, under the constraints (1), $x(0)=x_{0} \in \mathbb{R}^{n}$ and $x\left(t_{f}\right)=x_{f} \in \mathbb{R}^{n}$.

3 The symmetric case when $x(0)$ is quadratically weighted and $x\left(t_{f}\right)$ is assigned is similar. 
This case is obtained by the general one considered in Section 3 by assuming $\beta_{0}=\beta_{f}=0, W_{0}=W_{f}=I_{n}$, $x_{0}=w_{0}$ and $x_{f}=w_{f}$.

Since in this case $s=r=n$, the values of $p$ and $q$ providing the optimal solution are given by

$\left[\begin{array}{l}x_{0} \\ x_{f}\end{array}\right]=L\left[\begin{array}{l}p \\ q\end{array}\right]$

Notice that $L$ is non-singular by Lemma 1 , and $p$ and $q$ can be determined by matrix inversion.

The following result points out how these considerations enable important structural properties of the optimal solution to be derived in a direct and simple form.

Lemma 4 The optimal state, costate and input function for Problem 1a are unique, they are linear in $x_{0}$ and $x_{f}$ and they are given by

$x(t)=X_{1}(t) X_{1}^{-1}(0) x_{0}+X_{2}(t) X_{2}^{-1}\left(t_{f}\right) x_{f}$,

$\lambda(t)=\Lambda_{1}(t) X_{1}^{-1}(0) x_{0}+\Lambda_{2}(t) X_{2}^{-1}\left(t_{f}\right) x_{f}$,

$u(t)=U_{1}(t) X_{1}^{-1}(0) x_{0}+U_{2}(t) X_{2}^{-1}\left(t_{f}\right) x_{f}$,

where for all $t \in\left[0, t_{f}\right]$

$$
\begin{aligned}
& X_{1}(t):=e^{A_{+} t}-e^{A_{-}\left(t-t_{f}\right)} F_{+}, \\
& X_{2}(t):=e^{A_{-}\left(t-t_{f}\right)}-e^{A_{+} t} F_{-}^{-1}, \\
& \Lambda_{1}(t):=P_{+} e^{A_{+} t}-P_{-} e^{A_{-}\left(t-t_{f}\right)} F_{+}, \\
& \Lambda_{2}(t):=P_{-} e^{A_{-}\left(t-t_{f}\right)}-P_{+} e^{A_{+} t} F_{-}^{-1}, \\
& U_{1}(t):=-K_{+} e^{A_{+} t}-K_{-} e^{A_{-}\left(t-t_{f}\right)} F_{+}, \\
& U_{2}(t):=-K_{-} e^{A_{-}\left(t-t_{f}\right)}+K_{+} e^{A_{+} t} F_{-}^{-1} .
\end{aligned}
$$

The optimal cost is the quadratic form in $x_{0}$ and $x_{f}$

$J=\left[\begin{array}{ll}x_{0}^{\top} & x_{f}^{\top}\end{array}\right]\left[\begin{array}{cc}\Lambda_{1}(0) X_{1}^{-1}(0) & \Lambda_{2}(0) X_{2}^{-1}\left(t_{f}\right) \\ -\Lambda_{1}\left(t_{f}\right) X_{1}^{-1}(0) & -\Lambda_{2}\left(t_{f}\right) X_{2}^{-1}\left(t_{f}\right)\end{array}\right]\left[\begin{array}{c}x_{0} \\ x_{f}\end{array}\right]$

Proof: A straightforward computation, consisting of deriving $p$ and $q$ satisfying (34) and replacing them in (16), yields (35)-(37). Notice that $X_{1}(0)$ and $X_{2}\left(t_{f}\right)$ are non-singular, as their determinants equal that of $L$. Uniqueness of the optimal solution follows from the invertibility of $L$. The expression of the optimal cost is a direct consequence of Lemma 3.

Now we consider the classical version of the finitehorizon LQ problem, i.e., that in which the initial state is completely assigned and the terminal state is weighted quadratically in the performance index with $z_{f}=0$ without terminal constraints $(2)$.
Problem 1b Find a measurable input function $u(t)$, $t \in\left[0, t_{f}\right]$, and an absolutely continuous state trajectory $x(t), t \in\left[0, t_{f}\right]$, minimizing the quadratic performance index (3) with $\beta_{0}=0$ and $z_{f}=0$ under the constraints (1) and $x(0)=x_{0} \in \mathbb{R}^{n}$.

This case is obtained by the general one considered in Section 3 by assuming $W_{0}=I_{n}, w_{0}=x_{0}$ and $z_{f}=0$. In this case $s=n$ and $r=0$. Hence, the boundary condition (8) reduces to $\lambda\left(t_{f}\right)=P_{f} x\left(t_{f}\right)$ yielding by (16)

$\left(P_{f}-P_{+}\right) F_{+} p+\left(P_{f}-P_{-}\right) q=0$.

Equation (38), together with (16) evaluated at $t=0$, leads to the compact expression

$\left[\begin{array}{c}x_{0} \\ 0\end{array}\right]=L^{\prime}\left[\begin{array}{l}p \\ q\end{array}\right], L^{\prime}:=\left[\begin{array}{cc}I_{n} & F_{-}^{-1} \\ \left(P_{f}-P_{+}\right) F_{+} & P_{f}-P_{-}\end{array}\right]$.

It is not difficult to see that matrix $L^{\prime}$ is non-singular. Hence, by matrix inversion, equation (39) enables $p$ and $q$ to be computed.

Lemma 5 The optimal state, costate and input function for Problem $1 b$ are unique, they are linear in $x_{0}$, and they are given by

$$
\begin{aligned}
& x(t)=X(t) X^{-1}(0) x_{0} \\
& \lambda(t)=\Lambda(t) X^{-1}(0) x_{0} \\
& u(t)=U(t) X^{-1}(0) x_{0}
\end{aligned}
$$

where for all $t \in\left[0, t_{f}\right]$

$$
\begin{gathered}
X(t)=e^{A_{+}\left(t-t_{f}\right)} \Delta^{-1}\left(P_{f}-P_{-}\right) \\
+e^{A_{-}\left(t-t_{f}\right)} \Delta^{-1}\left(P_{+}-P_{f}\right) \\
\Lambda(t)=P_{+} e^{A_{+}\left(t-t_{f}\right)} \Delta^{-1}\left(P_{f}-P_{-}\right) \\
+P_{-} e^{A_{-}\left(t-t_{f}\right)} \Delta^{-1}\left(P_{+}-P_{f}\right), \\
U(t)=-K_{+} e^{A_{+}\left(t-t_{f}\right)} \Delta^{-1}\left(P_{f}-P_{-}\right) \\
-K_{-} e^{A_{-}\left(t-t_{f}\right)} \Delta^{-1}\left(P_{+}-P_{f}\right),
\end{gathered}
$$

where $\Delta:=P_{+}-P_{-}$. The optimal cost is the quadratic form $J(x, u)=x_{0}^{\top} \Lambda(0) X^{-1}(0) x_{0}$.

Proof: Note that $X\left(t_{f}\right)=I_{n}$ and $\Lambda\left(t_{f}\right)=P_{f}$. Then, $X(t)$ is non-singular for all $t \in\left[0, t_{f}\right]$ (see [5, Theorems 2 and 3$]$ ). Hence $X(0)$ is invertible. Relations (40)-(42) are derived by replacing the values of $p$ and $q$ satisfying (39) in (16) after simple algebraic manipulations. The optimal cost follows from Lemma 3.

\section{Concluding remarks}

A new methodology is developed for the solution of the finite-horizon LQ problem, in the general case where 
the initial and terminal states are subject to affine constraints and biased quadratic costs. This method consists of parametrizing all the solutions of the Hamiltonian differential equation in closed-form, thus avoiding the integration of the differential Riccati equation and taking advantage of standard and very efficient routines for the solution of algebraic Riccati equations.

This procedure easily provides the expressions for the optimal state and costate trajectories as well as the optimal control law in finite terms.

\section{References}

[1] L.D. Berkovitz. Optimal Control Theory. Springer-Verlag, New York, 1974.

[2] P. Brunovsky and J. Komornik. The Riccati equation solution of the linear quadratic problem with constrained terminal state. IEEE Transactions on Automatic Control, AC-26(2):398-402, 1981.

[3] F.M. Callier, J. Winkin, and J.L. Willems. Convergence of the time-invariant Riccati differential equation and LQproblem: mechanisms of attraction. International Journal of Control, 59(4):983-1000, 1994.

[4] L. Cesari. Optimization - theory and applications: problems with ordinary differential equations. Springer, New York, 1983.

[5] W.A. Coppel. Linear-quadratic optimal control. In Proceedings of the Royal Society of Edinburgh, volume 73A, pages 271-289, 1974.

[6] C.E.T. Dorea and B.E.A. Milani. Design of L-Q regulators for state constrained continuous-time systems. IEEE Transactions on Automatic Control, 40(3):544-548, 1995.

[7] J.N Juang, Turner J.D., and H.M. Chun. Closed-form solutions for a class of optimal quadratic regulator problems with terminal constraints. Transactions of the ASME, Journal of Dynamic Systems, Measurement, and Control, 108(1):44-48, 1986.

[8] A. Kojima and M. Morari. LQ control for constrained continuous-time systems. Automatica, 40(7):1143-1155, 2004.

[9] F.L. Lewis and V. Syrmos. Optimal Control. John Wiley \& Sons, New York, 1995.

[10] B.P. Molinari. The time-invariant linear-quadratic optimal control problem. Automatica, 13:347-357, 1977.

[11] H. Perez and S. Devasia. Optimal output-transitions for linear systems. Automatica, 39(2):181-192, 2003.

[12] A. Saberi, P. Sannuti, and B.M. Chen. $\mathrm{H}_{2}$ Optimal Control. System and Control Engineering. Prentice Hall International, London, 1995.

[13] P.O.M. Scokaert and J.B. Rawlings. Constrained linear quadratic regulation. IEEE Transactions on Automatic Control, AC-43(8):1163-1169, 1998.

[14] J.C. Willems. Least squares stationary optimal control and the algebraic Riccati equation. IEEE Transactions on Automatic Control, AC-16(6):621-634, 1971. 